\title{
Perspectives on learning styles in motor and sport skills
}

\author{
Ian Tobias Fuelscher ${ }^{1}$, Kevin Ball ${ }^{2}$ and Clare MacMahon ${ }^{2 *}$ \\ ${ }^{1}$ Department of Social Sciences and Psychology, Victoria University, Melbourne, VIC, Australia \\ ${ }^{2}$ Department of Sport and Exercise Science, Institute of Sport, Exercise and Active Living, Victoria University, Melbourne, VIC, Australia
}

\section{Edited by:}

Nicola Jane Hodges, University of

British Columbia, Canada

\section{Reviewed by:}

Markus Raab, German Sport

University Cologne, Germany

Paul Ford, Liverpool John Moores

University, UK

*Correspondence:

Clare MacMahon, Department of Sport and Exercise Science, Victoria

University, Ballarat Road, Footscray,

Melbourne, VIC 3011, Australia.

e-mail: clare.macmahon@vu.edu.au
We present the perspective that while coaches and instructors commonly adapt learning styles to maximize training outcomes, there has been little to no empirical support for the efficacy of this practice. Learning styles is a learner's preferred mode (e.g., visual, verbal) of taking in and processing new information. Although it is a relevant topic for the learning of motor and sport skills, few studies have used an appropriate methodology to test the effectiveness of learning style-based instruction. We highlight the need for a learning style assessment tool specific to motor skills and call for a test of the learning style hypothesis, the claim that learners will benefit from instruction that is tailored to their individual learning style. To this end, we suggest methodological guidelines.

\section{Keywords: learning styles, sport skills, tennis, skill acquisition, instruction}

Sport science is concerned with maximizing training outcomes, including the acceleration of learning and refinement for skilled performance. A factor in the rate of learning that has been identified in education, though not addressed in depth in motor learning, is learning style. Learning style is generally a learner's preferred ways of responding (cognitively and behaviorally) to learning tasks. It has been defined as a state-like learning preference, that is, a learning preference that changes depending on the learning environment or context. (Peterson et al., 2009) There is mounting evidence that learning styles are distinct from cognitive processing abilities. (Mayer and Massa, 2003)

While a detailed review of the available learning styles literature is beyond the scope of this article, current practices by classroom teachers and sports coaches highlight the relevance of learning styles for designing an effective learning environment. Borrowing form the available literature in educational and sport psychology, we suggest methodological guidelines to test the effectiveness of instructing athletes in ways that are consistent with their learning preference. During practice coaches commonly present technical cues to the athlete using different modalities (e.g., visual, verbal). This process requires athletes to take in and apply the new-learnt information. We propose technical skill development as a potential avenue for the application of learning styles to coaching and instruction. If learning styles-based instruction facilitates communication between coach and athlete, then it is reasonable to consider that athletes from different sports and with different levels of expertise might benefit from such instruction.

Over the past century, the learning style perspective has acquired great influence within the educational field. Pashler et al. (2009) note that there is a thriving industry devoted to publishing learning style tests and guidebooks for teachers. The authors further highlight that many organizations offer professional development workshops that are built around the concept of matching learning preferences/abilities and instructional methods. In support of these practices, Sternberg et al. (1999) for example found that college students showed improved learning when they were instructed in ways that were consistent with their ability patterns. For a full review on available learning style theories and models see Cassidy (2004).

Although it seems to be a relevant topic for the learning of motor skills, including sport skills, there is a lack of research investigating the relevance of learning styles to sport. This finding is not surprising, given the long held view that general motor ability (Ackerman, 1987; Adams, 1987) and task specific motor ability (Fleishman and Hempel, 1955) are one of the major determinants of skilled task performance. In the literature, motor ability (e.g., balance, timing) has been described as a relatively stable predictor of a person's achievement potential for skilled performance. (Magill, 2011) Consequently, studies investigating the relevance of individual difference variables for skill acquisition mostly considered the impact of stable performance predictors such as motor ability.

More recent perspectives in sport, however, emphasize the large impact that training (e.g., Ericsson et al., 1993) and the structure of training (e.g., Patterson and Lee, 2008) can have on success. In addition, a large number of professional sports teams have become engaged in learning styles testing (Schwab, 2011), acknowledging the potential role of learning style for learning progress (e.g., skill development) and learning outcomes (e.g., performance assessment). It is thus appropriate for sport scientists to examine the area of learning styles toward the goal of designing an effective training environment.

\section{CURRENT PERSPECTIVES}

A component of learning styles that has been adopted to assist coaches and instructors toward the goal of maximizing training is perceptual preference. Perceptual preference refers to an athlete's preferred sensory modality (e.g., verbal, visual) for receiving and attending to information in the environment. Given that athletes typically employ multiple senses during skill skilled performance, perceptual preferences can reasonably be expected to influence the learning process. 
A popular way of adapting this concept to coaching and instruction is to match perceptual preference and instructional method. These practices reflect more recent perspectives in sport, which acknowledge the role that individual difference variables (e.g., learning style) play for an effective communication process between coach and athlete (Reid et al., 2007).

While coaches and instructors commonly adopt learning styles to maximize training outcomes, and there is a theoretical base for the practice, there has been little to no empirical support for the efficacy of the practice to date. In an attempt to provide a methodological framework capable of assessing the effectiveness of learning style-based instruction, Pashler et al. (2009) defined and investigated the learning style hypothesis. This hypothesis claims that learners will benefit from instruction that is tailored to their individual learning style. According to Pashler and colleagues, credible validation of the learning style hypothesis requires robust documentation that the learning method that optimizes learning for one learning style group is different to the learning method that optimizes learning for a second learning style group. Following their review of the literature (primarily explaining traditional classroom-type tasks and learning) the authors found no studies that provided such evidence.

To address this issue, Pashler et al. (2009) suggested the following design, which can be applied to the sporting context: first, on the basis of a chosen measure of learning style, athletes should be divided into two or more groups (e.g., visual and verbal learners). Second, athletes within each learning style group are randomly assigned to one of at least two different learning methods (e.g., visual and verbal learning method). Third, following the instructional phase, all athletes are to be given the same test of achievement. As noted above, in order to support the learning style hypothesis, the results of this design need to show that the learning method that optimizes test performance in one learning style group is different to the learning method that optimizes test performance of a second learning style group.

Few studies have used an experimental methodology capable of assessing the effectiveness of learning style-based instruction on the basis of the above guidelines. Studies that investigated the application of learning preferences/abilities to instruction (e.g., Sternberg et al., 1999) generally assigned learners to either a 'matched' group (i.e., learners were instructed in a way that was consistent with their learning preference/ability) or an unmatched group. After the learning phase, all learners completed the same test of achievement. The analysis then compared the mean learning scores of both groups. The problem with this design however is that it cannot test whether the learning method that is best for one learning preference/ability group differs to the learning method that is best for a second learning preference/ability group.

Another gap in the available literature on learning styles for sport skills is that no empirically validated tools currently exist to assess learning styles in athletes. While numerous learning style tools exist to assess learning styles in the classroom (see Cassidy, 2004 for a review), the only inventory specific to motor skills is the VARK-athlete (Bonwell and Fleming, 2001). In recent times, the VARK-athlete has been the primary test used in sports settings to evaluate learning in motor skills (Schwab, 2011). However, the athlete version of the VARK is still based on classroom content learning and not based on learning motor skills. For example, a sample item is as follows:

You are about to give directions to an athlete who is standing with you. The athlete is new to town and needs to get to the athletic complex. The athlete has a bike. I would: a) draw a map on paper b) tell them the directions c) write down the directions d) bike with them to the complex (Bonwell and Fleming, 2001).

Rather than using an example of how the learner would prefer to learn a motor skill, as is the case for athletes, this question has been created by simply transferring the original classroom-setting question to a sporting environment. All 13 questions replicate the above example. Instead, an athlete learning styles questionnaire to evaluate preferred learning styles for motor skills should include items that are specific to motor skills in sport specific environments. Moreover, recent evidence suggests that validity of the VARK (and thus presumably the VARK-athlete) is questionable. (Leite et al., 2010)

\section{FUTURE DIRECTIONS}

With these shortcomings in mind, avenues for future research in the area of learning styles for sport skills are clear. First, future research should attempt to design and validate an assessment tool for learning styles in the sport environment. Second, in order to credibly assess the effectiveness of learning style-based instruction toward the goal of maximizing training, the learning style hypothesis needs to be empirically validated. Finally, future research should seek to reveal the mechanisms underlying perceptual preference and their acquisition.

While Pashler et al. (s' 2009) design provides a methodology capable of testing the learning style hypothesis, we further suggest supplementing it by including a test of skill proficiency at the beginning of the experiment as a potential covariate. This inclusion is made to control for the rate of skill acquisition during practice, which has been found to vary according to individual differences in skill proficiency between learners (Boyle and Ackerman, 2004). That is, athletes with lower skill proficiency at the beginning of the training phase can reasonably be expected to improve more than athletes with higher levels of skill, irrespective of instructional methods.

Two key components of Pashler's design will have to be addressed however before it can be implemented successfully: first, research must design and validate a sport specific assessment tool for learning styles in athletes. We define learning styles as learning preferences, rather than as cognitive abilities, which are likely to change depending on the learning context. In line with this definition, we recommend using questionnaires to identify athletes' preferred modalities across different sports (e.g., basketball, golf). Second, research should identify multiple measures to assess achievement or skill improvement in athletes. Depending on the sport, such measures could include form, performance, and transfer. In tennis for example, these measures would reflect technical stroke production, accuracy of the shot, and whether the athlete is able to apply the new-learnt information in a match situation. 
A possible avenue for testing the effectiveness of learning styles according to Pashler's design could be during technical skill development. In training, coaches commonly present new information visually (e.g., demonstrations) and verbally (explanations) to the athlete. To test the learning style hypothesis, athletes will have to be classified according to their learning style (e.g., visual or verbal as described above) and then be randomly assigned to a learning method (e.g., visual or verbal). During the intervention phase, the instructor will present technical cues to both learning groups that are designed to enhance skilled performance.

Both learning groups will be taught equivalent cues to ensure that the visual and verbal learning methods have the same impact to learn a movement. Athletes in the visual group will be taught via demonstrations only, while athletes in the verbal group will be taught via explanations only. After the learning phase, all athletes

\section{REFERENCES}

Ackerman, P. (1987). Individual difference in skill learning: an integration of psychometric and information processing perspectives. Psychol. Bull. 102, 3-27.

Adams, J. A. (1987). Historical review and appraisal of research on the learning, retention, and transfer of human motor skills. Psychol. Bull. 101, 41-74.

Bonwell, C. C., and Fleming, N. (2001). The VARK Questionnaire-Athletes Version. Available at: www.varklearn.com/documents/athletes.pdf [accessed November 6, 2011].

Boyle, M. O., and Ackerman, P. L. (2004). "Individual differences in skill acquisition," in Skill Acquisition in Sport, eds A. M. Williams and N. J. Hodge (New York: Routledge), 84-102.

Cassidy, S. (2004). Learning styles: an overview of theories, models, and measures. Educ. Psychol. 24, 419-444.

Ericsson, K. A., Krampe, R. T., and Tesch-Römer, C. (1993). The role of deliberate practice in the acquisition of expert performance. Psychol. Rev. 100, 363-406.

Fleishman, E. A., and Hempel, W. E. (1955). The relation between abilities and improvement with practice in a visual discrimination reaction task. J. Exp. Psychol. 49, 301-312.

Leite, W. L., Svinicki, M., and Shi, Y. (2010). Attempted validation of the scores of the vark: learning styles inventory with multitraitmultimethod confirmatory factor analysis models. Educ. Psychol. Meas. 70, 323-339.

Magill, R. A. (2011). Motor Learning and Control. New York: McGraw-Hill.

Mayer, R. E., and Massa, L. J. (2003). Three facets of visual and verbal learners: cognitive ability, cognitive style, and learning preference. J. Educ. Psychol. 95, 833-846.

Pashler, H., McDaniel, M., Rohrer, D., and Bjork, R. (2009). Learning styles: concepts and evidence. Psychol. Sci. Public Interest 9, 105-119.

complete the same test of achievement and/or skill improvement. Individual differences between athletes at the beginning of the experiment can be controlled for statistically by including skill proficiency as a covariate. A strength of this design therefore is that it can be applied across different sports and with athletes of different levels of expertise.

Together, these recommendations for future research highlight exciting avenues for investigating the application of learning styles to sport skills. Designing an assessment tool specific to motor skills and testing the learning style hypothesis for sport skills will be a first step toward evaluating the effectiveness of learning style-based instruction for athletes. Identifying how athletes prefer to learn could assist coaches when introducing new skills or fine-tuning established ones, thus improving outcomes and performance. This in turn could accelerate the learning of these skills and therefore improve performance of the athletes.

Patterson, J. T., and Lee, T. D. (2008). Examining the proactive and retroactive placement of augmented information for learning a novel computer alphabet. Can. J. Exp. Psychol. 62, 42-50.

Peterson, E. R., Rayner, S. G., and Armstrong, S. J. (2009). Herding Cats: In Search of Definitions of Cognitive Styles and Learning Styles. ELSIN Newsletter, Winter 2008-2009. Available at: www.elsinnews.com [accessed December 22, 2011].

Reid, M., Crespo, M., Lay, B., and Berry, J. (2007). Skill acquisition in tennis: research and current practice. J. Sci. Med. Sport 10, 1-10.

Schwab, P. (2011). The Coach as a Teacher. Available at: www.afl.com. au/news/newsarticle/tabid/208/news id/121159/default.aspx [accessed November 06, 2011].

Sternberg, R. J., Grigorenko, E. L., Ferrari, M., and Clinkenbeard, P. (1999). A triarchic analysis of an aptitude-treatment interaction. Eur. J. Psychol. Assess. 15, 1-11.
Conflict of Interest Statement: The authors declare that the research was conducted in the absence of any commercial or financial relationships that could be construed as a potential conflict of interest.

Received: 16 November 2011; paper pending published: 28 November 2011; accepted: 21 February 2012; published online: 07 March 2012.

Citation: Fuelscher IT, Ball $K$ and MacMahon C (2012) Perspectives on learning styles in motor and sport skills. Front. Psychology 3:69. doi: 10.3389/fpsyg.2012.00069

This article was submitted to Frontiers in Movement Science and Sport Psychology, a specialty of Frontiers in Psychology. Copyright (c) 2012 Fuelscher, Ball and MacMahon. This is an open-access article distributed under the terms of the Creative Commons Attribution Non Commercial License, which permits noncommercial use, distribution, and reproduction in other forums, provided the original authors and source are credited. 